\title{
Novel CNTs grafting on carbon fibres through CVD: investigation of epoxy matrix/fibre interface via nanoindentation
}

\author{
Stefania Termine Aikaterini-Flora A. Trompeta, Dimitrios A. Dragatogiannis, and Costas A. \\ Charitidis* \\ Research Lab of Advanced, Composite, Nanomaterials and Nanotechnology (R-NanoLab), School of \\ Chemical Engineering, National Technical University of Athens, 9 Heroon Polytechniou str., \\ Zographos, 15780, Athens, Greece, charitidis@chemeng.ntua.gr*
}

\begin{abstract}
Carbon Fibre Reinforced Polymers (CFRPs) have spread to a broad range of sectors including automobile, aeronautics and space industry the last decades. Recently, the emergence of new requirements for improved smart properties and functionalities have been main drivers to the introduction of novel methodologies and optimization of processes. A new approach of functionalizing CFs is the in-situ grafting of carbon nanotubes (CNTs) onto the surface of fibres, through chemical vapour deposition (CVD). In this study, CFRPs were manufactured via Vacuum Assisted Resin Transfer Molding (VARTM) and characterized by microscopy techniques through their cross-section. The effect of CNTs in-situ grafting onto the CFs on the mechanical behavior of the composite was studied both in micro- and macro-scale level, through instrumented indentation technique and tensile testing. The mechanical behaviour of the composite with the CNTs in-situ grafted onto the CFs was compared with CNTs-modified composites, containing CNTs in the epoxy matrix. Comparing the nanomechanical properties with conventional mechanical testing, the enhancement of mechanical behaviour was revealed for the case of the CNTs-modified composite. Additionally, an increased interfacial adhesion between the CNTs-functionalised CFs and the polymer matrix was observed, indicating that CNTs contribute to an enhanced bonding between matrix and CFs.
\end{abstract}

\section{Introduction}

Composite materials and specifically carbon fibre reinforced polymers (CFRPs), play a significant role as structural aerospace components [1], due to their exceptional strength and stiffness-to-density/weight ratio. Additionally, their strength and stiffness can be tailored according to the desirable direction. CFRPs are capable to be manufactured in large and complex shapes, while maintaining shape and mechanical integrity in aeronautics and space environment.

Despite the fact that CFRPs present high resistance to fatigue and environmental corrosion, they suffer from their brittleness and low impact strength. This may cause internal damage, such as cracks, fibre breakage, fibre debonding or delamination [2]. Depending also on the polymer matrix used, moisture absorption can deteriorate the composite. This comes 
together with the high temperature variations that are met in this kind of environment, which can cause severe degradation [3]. Finally, a common drawback is the weak interface between matrix and reinforcement (e.g. carbon fibres -CFs), which results in low tensile strength in out-of-plane loads and poor stress transfer between fibre and matrix [4].

Recent investigations focus on the functionalization of the CF surface by various techniques, including electropolymerisation [5-7], electrophoretic deposition (EPD) [8] and chemical vapour deposition (CVD) [9]. Many researchers have focused on the in-situ growth of carbon nanotubes (CNTs) or CNTs deposition onto the CF surface, due to their potential to increase significant load bearing capacity of composites; whilst overcoming the critical problems that usually encountered in the composite fabrication process, such as, agglomeration and degradation of the CNTs [10]. Comparing CVD with EPD, it turns out that growing CNTs onto the CF surface instead of attaching them, enables the control of size, quality and quantity, as well as alignment of CNTs on surface of CF. The CNT grafting to CFs was proved to increase adhesion of CF with matrix, which results in a good transfer of electrical charge and heat between the nanotubes and fibres [11]. Finally, the mechanical properties of the nanocomposites were found to increase by increasing amount of deposited CNTs. Mathur et al. [12] in the study proved that the flexural strength (FS) improved by $20 \%$ for uni-directional, $75 \%$ for $2 \mathrm{D}$ and $66 \%$ for $3 \mathrm{D}$ hybrid composites as compared to that prepared by neat reinforcements (without CNT growth) under same conditions. Flexural modulus of the composites was also improved by $28 \%, 54 \%$ and $46 \%$, respectively.

Growing CNTs on the CF surface has advantages in terms of quantity, length, orientation and size of CNTs, regarding the obtained surface properties. However, still from the early studies of CNTs growth on CFs, the importance of preventing mechanical degradation of the CFs due to intension heating using standard CVD techniques was a major issue [13-14]. The excessive strength loss of reinforcing fibers can lead to a premature failure of composites under loadings and seriously affect the ultimate mechanical performance of composites [1516]. For this reason, CNTs are also utilized to modify the polymer matrix and by this to increase the cohesion of composites, while they offer also resistivity to the crack propagation. Also, CNTs improve the flexural strength of the composite, offering improved reinforcement and minimizing delamination and intra-ply cracks. Finally, void distribution in CFRPs, could be significant reduced, due to the presence of CNTs.

The motivation of this work was to study the effect of CNTs incorporation in CFRPs on the CF-matrix interface properties. For this reason, two routes were investigated; the in-situ growth of CNTs on the CF surface via the CVD method (modification of CF surface properties) and the addition of CNTs in the epoxy matrix (modification of polymer matrix). Nanoindentation was applied as a tool for studying the local mechanical behaviour of the fibre/matrix interface and worked complementary with the macromechanical testing and the visualisation of the interface through scanning electron microscopy.

\section{Experimental Procedure}

\subsection{CNTs growth on CFs via CVD}

G0926 CF fabrics (Hexcel Industries Inc., USA) were used as a substrate to graft CNTs on their surface. The grafting was achieved through the CVD process, by utilizing the floating catalyst approach, where the catalyst is introduced into the system by evaporation of a liquid hydrocarbon-based solution that contains the catalyst compound. The precursor solution consisted of ferrocene, dissolved through sonication in ethanol at the concentration of $2 \mathrm{wt}$. $\%$. The solution was introduced in a separating funnel and while feeding, it was evaporated 
by a heating mantle and carried by argon to the isothermal zone of the reactor. A cylindrical stainless-steel reactor in pilot scale was used, $(1.10 \mathrm{~m}$ length, $7 \mathrm{~cm}$ inner diameter, isothermal area of $40 \mathrm{~cm}$ ). The detailed experimental procedure is presented in the flow chart shown in Fig. 1.

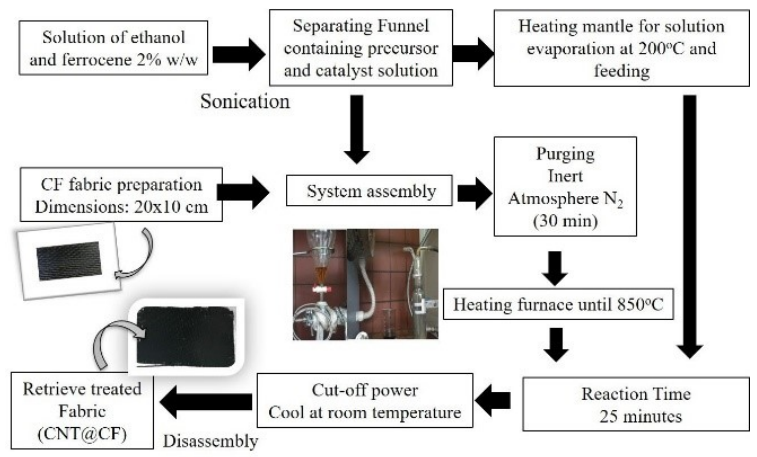

Fig. 1. Experimental procedure of CNTs growth on CF fabrics via the floating catalyst CVD method.

\subsection{CNTs incorporation in epoxy resin}

A hot curing epoxy matrix system (HUNTSMAN Industries, USA), with a mix ratio of 10090-0.5 parts per weight, was used as matrix. Functionalized CNTs with carboxyl groups were dispersed in the epoxy resin $(0.5 \mathrm{wt} . \%)$ in order to manufacture CFRPs with CNTs-modified matrix. In Fig. 2, a schematic representation of the experimental procedure for the dispersion of the CNTs in the epoxy system is shown. The first step was to de-agglomerate the CNTs by dispersing them in acetone, form a slurry and then sonicate it in ultra-sonic bath for 30 min. In the next step, the paste was introduced to the hardener component and shear mixed for $1 \mathrm{~h}$ with gradually increasing rotation speed (from 700 to $1000 \mathrm{rpm}$ ). After this stage and before introducing the other parts of the epoxy system, the mixture was sonicated for $30 \mathrm{~min}$. Finally, the epoxy monomer and the accelerator were added and then shear mixed in same mixing conditions.

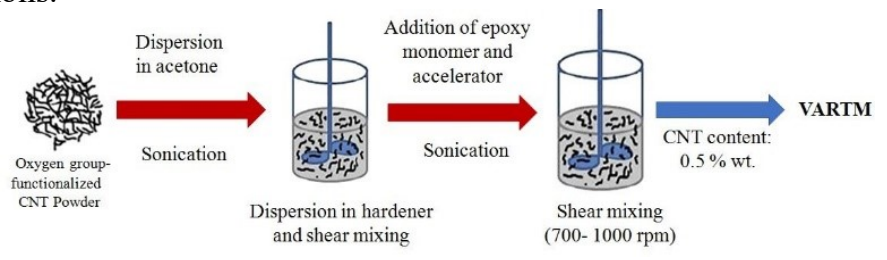

Fig. 2. Experimental procedure for CNTs addition in epoxy resin.

\subsection{Composites manufacturing}

All composites were manufactured through Vacuum Assisted Resin Transfer Molding (VARTM) process in room temperature. The CF fabric used was G0926 (Hexcel Industries Inc., USA) with plain 0/90 wave. Each fabric ply was placed in a panel-like type metal mold (apparatus shown in Fig. 3). Before the infusion, the epoxy system was heated at $50^{\circ} \mathrm{C}$ for 30 min to reduce its viscosity and then was de-gassed in vacuum. In Fig.4, the infusion profile of the resin through the fabrics during VARTM (vacuum: $10^{-3} \mathrm{mmHg}$ ) is shown. The final composite geometry is in panel form and from this, with a CNC waterjet cutting process, is possible to receive composite specimens of the desired dimensions for mechanical testing. 


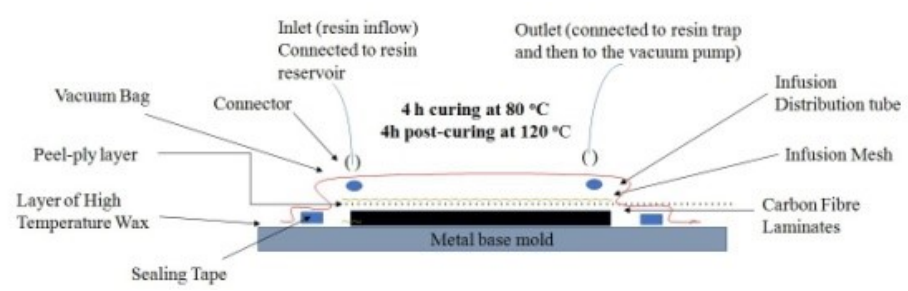

Fig. 3. Vacuum Assisted Resin Transfer Molding (VARTM) method.
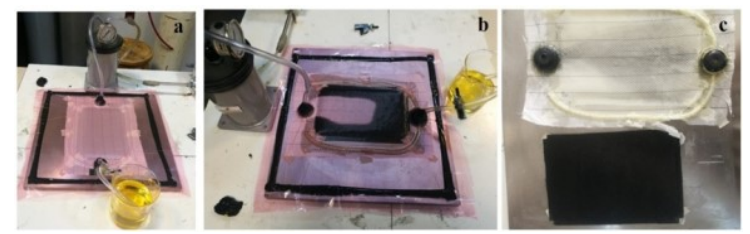

Fig. 4. Composites manufacturing steps: a) System under vacuum, b) resin propagation, c) Final composite (panel-type).

In Fig. 5, a schematic illustration of the cross-section of the three different CFRPs that were manufactured is presented. On the left is the pristine specimen (REF), in the middle the specimen with the CNTs dispersed in the epoxy matrix (CNT@EP) and on the right the specimen with the CNTs grafted on the CF surface (CNT@CF). It is worth noting, that in the case of the CNT@CF composite there is no polymer sizing on the CF (presented with red line). As the fabric is thermally treated during CVD, the commercial epoxy sizing is removed, as it also confirmed by other studies [17]. As a result, the adhesion between the fibre and the matrix in this case, depends only from the presence of the CNTs. Regarding the way that CNTs are oriented in the two modified composites, it may be remarked that in the case of the CNT@CF, CNTs are located radically around the surface of a single fibre (for the fibres that are on the surface of the fabric). On the other hand, regarding the composite with the CNTs dispersed into the matrix, the distribution and location of CNTs is hard to be determined, while it depends by the dispersion degree, the resin transfer and the wettability of the fibres. CNTs in this case are randomly oriented within the polymer that surrounds the CFs.

(a) REF

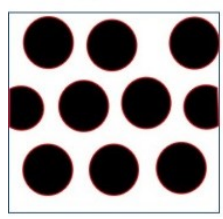

(b) CNT@EP

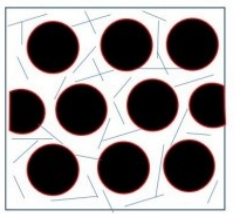

(c) CNT@CF

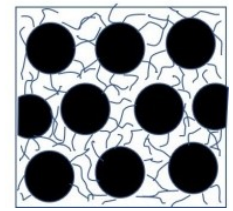

Fig. 5. Schematic illustration of CFRPs cross-section. Fibre sizing (red) and CNTs orientation (blue):

a) Reference CFRP, b) CNTs dispersed in epoxy matrix, c) CNTs grafted on CF surface.

\subsection{Characterization}

\subsubsection{Tensile testing}

Tensile tests were performed by Instron Tensile machine (MTS systems Co, USA) with a maximum load capacity of $100 \mathrm{kN}$. The testing was performed according to the ASTM D63802 standard. Three specimens of each sample in a dog-bone shape were packed to the machine gripers and uniaxial force was applied, with increasing rate. Microstrain was measured during loading by an external extensometer. 


\subsubsection{Nanoindentation testing}

A Hysitron Tribolab ${ }^{\circledR}$ Instrument, equipped with a standard three-sided pyramidal Berkovich tip, was used for the nanomechanical tests, at a load range from $1 \mu \mathrm{N}$ to $30 \mathrm{mN}$ with simultaneous recording of the displacement. A standard fused silica sample was used to calibrate the area function of the Berkovich indenter tip prior measuring [18-19].

\subsubsection{Scanning microscopy}

The morphology of the grown carbon nanostructures on the surface of the fabrics and the fractured area of the composites from the tensile testing, were characterized by Scanning Electron Microscopy-SEM (Hitachi TM3030 tabletop microscope). The capability of obtaining high magnification by operating at $5 \mathrm{kV}$, allows the observation of surface details such as traditional topographic imaging. Its magnification ability extends up to $\times 6000$.

\section{Results and Discussion}

\subsection{Assessment of CNTs growth on CF fabrics}

The morphology of the carbonaceous material grown on the surface of the CFs is presented in Fig.6. From the low magnification of $\times 100$, it can be seen that the growth is uniform on the surface fibres, but in the internal area of the bundle no deposition took place. In higher magnification of the surface fibres, it can be observed that CNTs growth took place not only on the upper surface of a fibre, but around the perimeter of every single fibre. Furthermore, in our case, no surface degradation of fibres was observed, that could be resulted from the thermal treatment. Further analysis of the structure of nanoparticles and the interface was carried out by TEM characterization, in Brightfield mode and magnification at $\times 440 \mathrm{k}$. Successful grafting of CNTs can be reported, where the catalytic nanoparticles have been embedded on the surface of the fibre and growth of bamboo-like CNTs structures took place, following the bottom up growth mechanism.

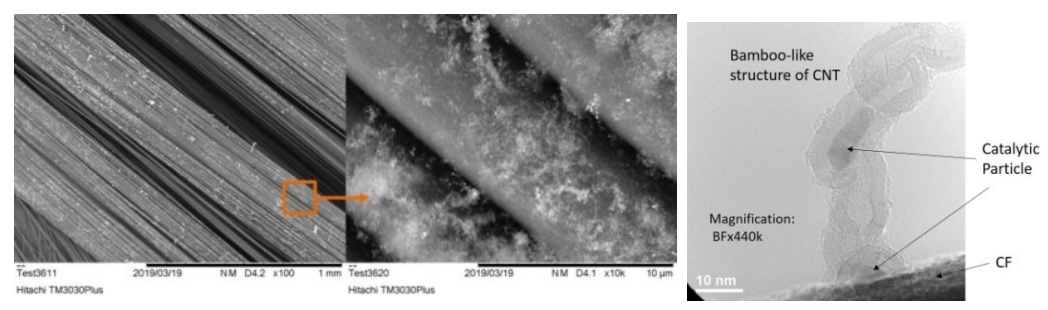

Fig. 6. SEM and TEM analysis of the CNTs grown on CF fibres.

\subsection{Assessment of CNTs dispersion in epoxy matrix}

A sample from the epoxy resin with CNTs after curing was studied under SEM, and the results are shown in Fig. 7. A homogenous dispersion can be noticed, since CNTs are covering the whole area of the cross-section, even in low magnification, without the formation of agglomerates. It may be remarked that the diameters of CNTs inside the composite were slightly increased (MWCNTs seem to become thicker). This indicates that the polymer has been wrapped around CNTs with non-covalent bonds. 

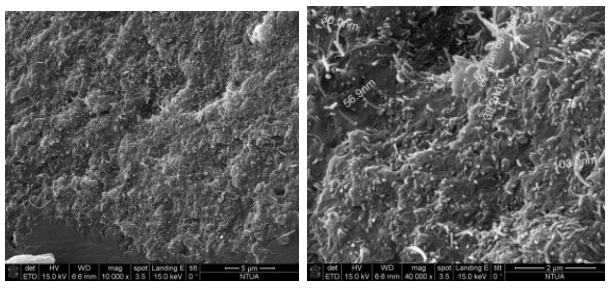

Fig. 7. SEM analysis in the CNT-nanocomposite for dispersion homogeneity assessment.

\subsection{Mechanical testing results}

\subsubsection{Tensile testing}

The results of the tensile testing showed that the composite with CNT-modified epoxy matrix, exhibited improvement in ultimate tensile strength, elongation and Young's Modulus, in comparison to the reference, by $5.81 \%, 1.90 \%$ and $1.58 \%$, respectively. On the other hand, CNTs@CF sample, demonstrated lower mechanical properties, by $42 \%$ in ultimate strength, $26 \%$ in elongation and $23.3 \%$ in elastic modulus, in respect to the reference one. The average of the actual values from the test, are shown in Table 1.

Table 1. Tensile test results.

\begin{tabular}{|c|c|c|c|}
\hline & Ultimate Tensile Strength (MPa) & Elongation (\%) & Tensile Modulus (MPa) \\
\hline REF & $646.7 \pm 30.1$ & $1.0 \pm 0.1$ & $63.1 \pm 1.9$ \\
\hline CNTs@CF & $371.0 \pm 35.0$ & $0.8 \pm 0.1$ & $48.4 \pm 1.4$ \\
\hline CNTs@EP & $684.3 \pm 27.1$ & $1.1 \pm 0.1$ & $64.1 \pm 1.9$ \\
\hline
\end{tabular}

\subsubsection{Nanoindentation testing}

Nanoindentation load-depth curves obtained in matrix and CF area, are shown in Fig. 8a and Fig. 8b, respectively. In Fig. 8b it is depicted that both samples CNT@CF and CNT@EP demonstrated higher resistance to applied nanoindentation load compared to REF. This is an indication of an increase of local stiffness, attributed to the improvement of CF-matrix adhesion and enhancement of interface comparing with REF. $\mathrm{E}_{\mathrm{r}}$ and $\mathrm{H}$ values obtained by indentations made on both CFs-matrix interface and epoxy matrix areas of the composite are summarized in Table 2. As seen, CNT modification of epoxy matrix resulted to improvement of both $\mathrm{E}_{\mathrm{r}}$ and $\mathrm{H}$ of composite, specifically in the CFs-matrix interface in comparison to insitu grafting of CNTs onto CF. $\mathrm{E}_{\mathrm{r}}$ values obtained by indentations that made exclusively in $\mathrm{CFs}$, were found slightly higher, comparing to mean $\mathrm{E}_{\mathrm{r}}$ values of the composites, indicating improvement of CF-matrix adhesion.
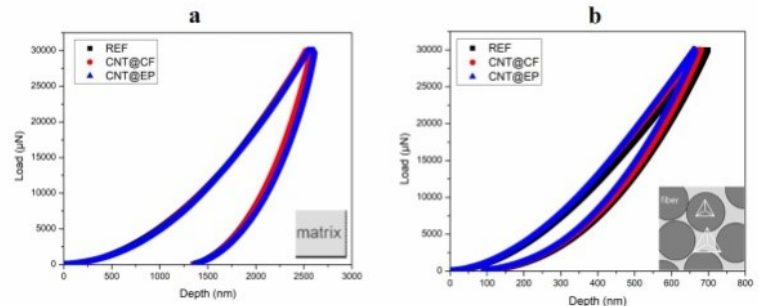

Fig. 8. Load-displacement curves obtained on each of the samples for nanoindentations made a) in the matrix and $b$ ) in the fiber area. 
Table 2. Er and $\mathrm{H}$ values obtained by indentations made on $\mathrm{CFs}$-matrix interface.

\begin{tabular}{|c|c|c|}
\hline Sample & Er (GPa) & H (GPa) \\
\hline REF & $52.4 \pm 8.3$ & $5.9 \pm 3.2$ \\
\hline CNT@CF & $52.3 \pm 7.2$ & $5.7 \pm 3.0$ \\
\hline CNT@EP & $63 . \pm 9.5$ & $7.8 \pm 3.9$ \\
\hline
\end{tabular}

\subsection{Study of the cross-section}

The cross-section images of the fractured composite specimens after tensile testing are presented in Fig. 9. For the REF composite, phenomena such as delamination and fibre pullout can be observed at the cross-section. In the $0^{\circ}$ direction, fiber breakage occurred while matrix has been removed from the intense fracture. Also, a large number of fibres that have been pulled-out from the surrounding matrix can be observed. In the $90^{\circ}$ direction, delamination occurred and fibre/fibre splitting is detected. In CNTs@EP composite, the morphology of the fracture is different. The matrix's texture is rough and irregular. The modified resin has not been removed and the fracture surface is flatter in comparison to the reference. Also, delamination phenomena were reduced but in $0^{\circ}$ direction some fibres have been pulled-out, but in total it could be mentioned that the matrix holds the fibres, that is an indication of good fibre/matrix adhesion. For the CNTs@CF composite, the interaction of the matrix/fibre is enhanced comparing to CNTs@EP. The resin is completely adhered to the fibres and the fracture in the $0^{\circ}$ direction appears in tufts. All fibres were coated with resin and cracking/breakage occurred only in the pure epoxy.
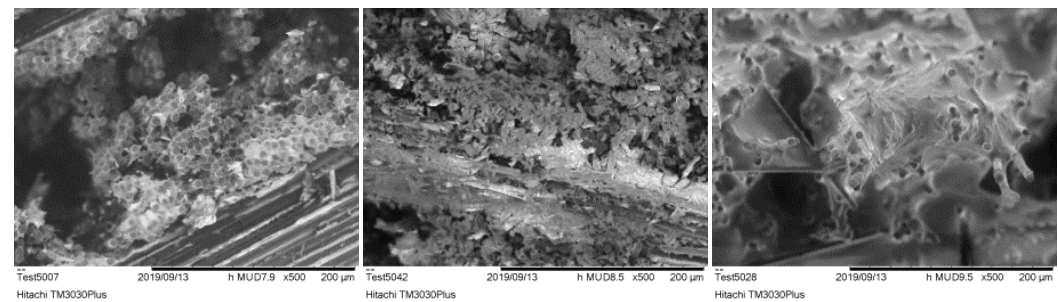

Fig. 9. SEM of CFRPs cross-section after tensile: Left REF, Middle CNTs@EP, Right CNTs@CF.

\section{Conclusions}

CNTs grafting on CF fabrics was achieved through CVD by utilizing a novel precursor/catalyst feeding system, taking into account "green" chemistry perspectives. By the developed approach, pilot scale deposition of CNTs on CF fabrics has proved feasible, which now enables manufacturing of CNTs-grafted CFRPs. Furthermore, two modified CFRPs, each of them presented different reinforcement type, were manufactured and evaluated in terms of their mechanical response at macro- and nanoscale level. The assessment of macro- and nano- mechanical behavior of the composite with the CNTs dispersed in the matrix, confirmed that $0.5 \mathrm{wt}$ \% CNTs contribute and improve the mechanical properties by $5.81 \%$ in ultimate strength. CNT modification of epoxy matrix resulted to improvement of both $\mathrm{E}_{\mathrm{r}}$ and $\mathrm{H}$ of composite, specifically in the CFs-matrix interface in comparison to other samples. Despite the fact that a reduction of mechanical properties in macroscale was observed for the composites with CNTs grown on CFs, nanoindentation results proved constant local mechanical properties similar to the pristine composite. Thus, any macromechanical failure could be attributed to artifacts during 
composite manufacturing. By exploiting carbon based nanomaterials, novel advanced multifunctional composites can be manufactured for various demanding applications.

This study was equally supported by the H2020 Projects: "Modified Cost-Effective Fibre Based Structures with Improved Multi-Functionality and Performance - MODCOMP" (GA No. 685844) and "Smart by Design and Intelligent by Architecture for turbine blade fan and structural components systems - SMARTFAN" (GA No. 760779).

\section{References}

1. A. Quilter (IHS ESDU, Englewood, USA, 2017)

2. S. Houston (Aviation, Aircraft, 2009)

3. R. Verma, (Intern. Department at OLYMSPAN, Autoclave \& Composite Manufacturing, 2018)

4. Z. Yuanxin P. Farhana, J. Shaik, M. Puspanjali, J. Mater. Process. Technol. 198 (2008)

5. D. Semitekolos, P. Goulis, D. Batsouli, E.P. Koumoulos, L. Zoumpoulakis, C.A. Charitidis, Int. J. Struct. Integ. (2019)

6. D. Semitekolos, P. Kainourgios, C. Jones, A. Rana, E.P. Koumoulos, C.A. Charitidis, Comp. Part B-Eng. 155, 237-243 (2018)

7. P. Kainourgios, I.A. Kartsonakis, D.A. Dragatogiannis, E.P. Koumoulos, P. Goulis, C.A. Charitidis, App. Surf. Sci. 416, 593-604 (2017)

8. J. Jiang, C. Xu, Y. Su, Q. Guo, F. Liu, C. Deng, X. Yao, L. Zhou, Polymers (Basel), 8, 302 (2016)

9. J. Zhang (Materials, Ecole Centrale, Paris, 2012)

10. M. Delmas, M. Pinault, S. Patel, D. Porterat, C. Reynaud, M. Mayne-L'Hermite, Nanotech. 23, 105604 (2012)

11. W.B. Down, R.T.K. Baker, JMR, 10, 625-633 (1995)

12. R.B. Mathura, S. Chatterje, B.P. Singh, Comp. Sci. Technol. 68, 1608-1615 (2008)

13. H. Qian, A. Bismarck, E.S. Greenhalgh, G. Kalinka, M.S.P. Shaffer, Chem. Mater. 20, 1862-1869 (2008)

14. J. Zhao, Liu L, Guo Q, Shi J, Zhai G, Song J, Carbon. 46, 380-3 (2008)

15. L. Feng, K.Z. Li, J.H. Lu, L.H. Qi, J, J. Mater. Sci. Technol. 33, 66-70 (2017)

16. N. De Greef, Zhang, A. Magrez, L. Forró, J.P. Locquet, I. Verpoest J.W. Seo, Diam \& Rel. Mater., 51, 39-48 (2015)

17. J.N. Coleman, Khan, W.J. Blau, Y.K. Gun'ko, Carbon 44 1624-1652 (2006)

18. P. Kavouras, D.A. Dragatogiannis, D.I. Batsouli, C.A. Charitidis, Polym. Test. 61, 197-204 (2017)

19. Z. Zhang, S.C. Gallo, X. Li, H. Dong, D. Dragatogiannis, C.A. Charitidis, Polym. Test. 80106091 (2019) 